\title{
Skyrmion domain wall collision and domain wall-gated skyrmion logic
}

\author{
Xiangjun Xing, ${ }^{1,2}$ Philip W. T. Pong, ${ }^{1}$ and Yan Zhou ${ }^{3,4, *}$ \\ ${ }^{1}$ Department of Electrical and Electronic Engineering, The University of Hong Kong, Hong Kong, China \\ ${ }^{2}$ College of Physics and Electronic Information Engineering, Wenzhou University, Wenzhou 325035, China \\ ${ }^{3}$ Department of Physics, The University of Hong Kong, Hong Kong, China \\ ${ }^{4}$ School of Electronic Science and Engineering and Collaborative Innovation Center of Advanced Microstructures, Nanjing University, \\ Nanjing 210093, China
}

(Received 20 April 2016; revised manuscript received 10 July 2016; published 4 August 2016)

\begin{abstract}
Skyrmions and domain walls are significant spin textures of great technological relevance to magnetic memory and logic applications, where they can be used as carriers of information. The unique topology of skyrmions makes them display emergent dynamical properties as compared with domain walls. Some studies have demonstrated that the two topologically inequivalent magnetic objects could be interconverted by using cleverly designed geometric structures. Here, we numerically address the skyrmion domain wall collision in a magnetic racetrack by introducing relative motion between the two objects based on a specially designed junction. An electric current serves as the driving force that moves a skyrmion toward a trapped domain wall pair. We see different types of collision dynamics depending on the driving parameters. Most importantly, the modulation of skyrmion transport using domain walls is realized in this system, allowing a set of domain wall-gated logical NOT, NAND, and NOR gates to be constructed. This work provides a skyrmion-based spin-logic architecture that is fully compatible with racetrack memories.
\end{abstract}

DOI: 10.1103/PhysRevB.94.054408

\section{INTRODUCTION}

Skyrmions [1-3] and chiral domain walls (DWs) [4,5] are two different classes of (meta)stable spin configurations in ultrathin magnetic multilayer nanostructures, where the interfacial Dzyaloshinskii-Moriya interaction (DMI) [6-8]induced conjunctly by the broken inversion symmetry of a ferromagnetic layer and the strong spin-orbit coupling in a heavymetal layer [9] — tends to twist adjacent magnetic moments and relate a specific chirality to the induced spin textures. Skyrmions-firstly postulated by Skyrme in a continuous field theory to describe baryon stability [10] and subsequently observed in a variety of physical systems [11,12] — carry a unit topological charge and thus belong to topologically protected structures; that is, they cannot be unwound via continuous deformation [13], which allows them to readily move under an ultralow current density [14-17] and to flexibly avoid pinning centers during motion [18-20]. Apart from these intriguing features, it was recognized that moving magnetic skyrmions can bring about an emergent electromagnetic field [15], and most recently, it was demonstrated that magnetic skyrmions can even be controllably written and erased using a tiny mechanical force exerted by a microtip [21]. Because of these merits, magnetic skyrmions serve as an active platform for researching new phenomena [20,22-25] and more importantly for building functional devices [18,26-33]. Compared to conventional DWs, chiral DWs show greatly enhanced mobility owing to the combined action of the DMI and spin Hall effect, and thus hold great promise for implementing new families of spintronic devices [34-37].

Up to now, most proposed examples of racetrack memory and logic devices employed skyrmions or DWs as information carriers [18,26-28,32,33]; few devices have been

\footnotetext{
*Author to whom correspondence should be addresed: yanzhou@hku.hk
}

exploited which take advantage of potential interplays between skyrmions and DWs for functional operations. As revealed in Ref. [38], when two skyrmions approach each other, they will shrink in size as a result of mutual repulsion. Furthermore, Iwasaki et al. studied the skyrmion-skyrmion collision in the $\mathrm{MnSi}$ system and found that the scattering process was almost elastic [39]. The DW-DW collision in soft magnetic nanotracks has also been studied and the collision outcome depended on the relative alignments of colliding DWs [40]. In a previous work [29], Zhou et al. proposed a hybrid racetrack memory based on coexisting skyrmions and DWs, where the skyrmions, directly used for functional operation, are converted from chiral DW pairs through a lateral junction. We now wonder what will happen when a skyrmion collides with a chiral DW. To this end, we need to find, first of all, a system that allows a skyrmion to approach a magnetic DW because no such system has yet been established, although the static structures and dynamic properties of skyrmions and chiral DWs have been well understood $[4,13,37,41-43]$ and the coexistence of these objects in a chiral magnet has been demonstrated $[29,44]$.

According to $v_{x}^{d} \propto(\beta / \alpha) J[19,39,45]$ (which approximately holds true for a skyrmion steadily flowing along a magnetic racetrack without defects, where $v_{x}^{d}$ is the skyrmion drift velocity along the long axis, $\alpha$ is the damping constant of the used magnetic material, $\beta$ is the ratio of the nonadiabatic and adiabatic spin torques, and $J$ is the current density), one expects higher skyrmion velocities in a given nanotrack simply by increasing the current density. To render a collision, there must be relative motion between a skyrmion and a DW pair, which can in principle be achieved by keeping one of them moving while the other is at rest. Based on the previous reports $[19,46-49]$, it is easier to pin a DW than to pin a skyrmion using geometric defects because DWs are spatially extended objects while skyrmions are localized ones. As such, we design an $\mathrm{H}$-shaped junction with four antinotches [Fig. 1(a)] to trap a DW pair [Figs. 1(b) and 2] via pinning effect [46,47]. We choose an electric current to drive the skyrmion and explore 
(a)
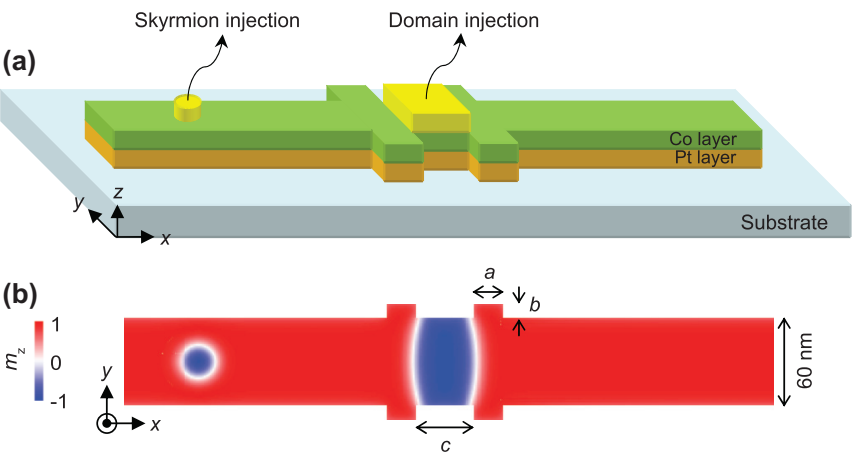

FIG. 1. Sketch of device structure. (a) Perspective view of the magnetic racetrack with an $\mathrm{H}$-shaped junction. Skyrmions and DWs can be injected into the nanotrack using nanocontact spin valves situated at the yellow elements. (b) A DW pair can be trapped in the junction, when a moderate current is applied to the nanotrack, whereas a skyrmion can freely flow along the nanotrack and gradually approach the DW, resulting in a skyrmion DW collision. The skyrmion DW repulsion together with current-induced torque can trigger complex collision dynamics. The nanotrack is $60 \mathrm{~nm}$ wide and $1 \mathrm{~nm}$ thick. The junction sizes $a, b$, and $c$ are 20,10, and $30 \mathrm{~nm}$, respectively.
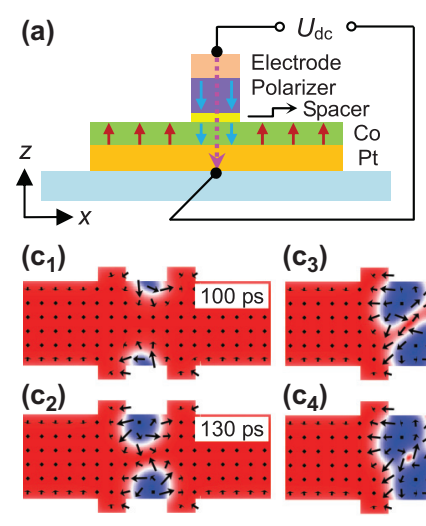

(d)

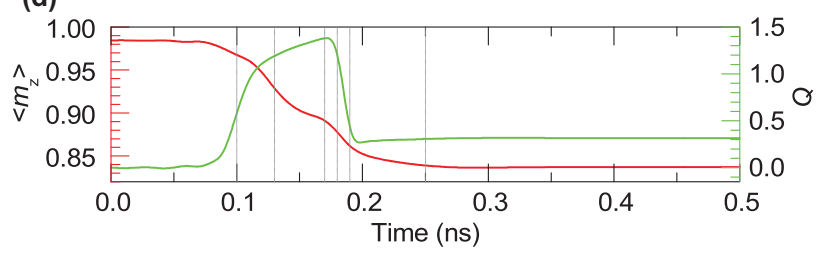

(e)

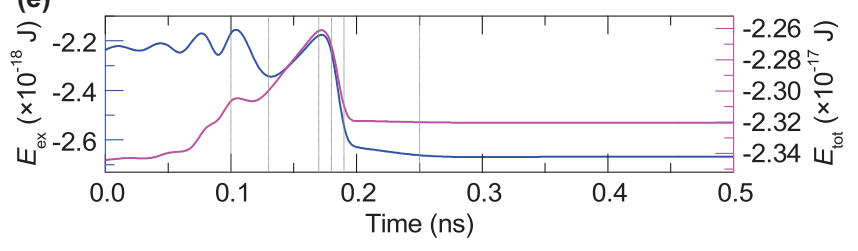

FIG. 2. Writing a DW pair into the junction. (a) Structure of nanocontact spin valve. (b) Initial state of the nanotrack. The yellow box encircles the current-action area. (c) Injection process for the DW pair. Current-action time is specified in each panel. (d) Topological charge $Q$ and vertical magnetization $m_{\mathrm{z}}$, averaged over the entire volume of the nanotrack, against current-action time. (e) Exchange energy $E_{\text {ex }}$ and total energy $E_{\text {tot }}$ against current-action time. The vertical dashed lines in $(\mathrm{d}, \mathrm{e})$ mark the temporal moments specified in (c).
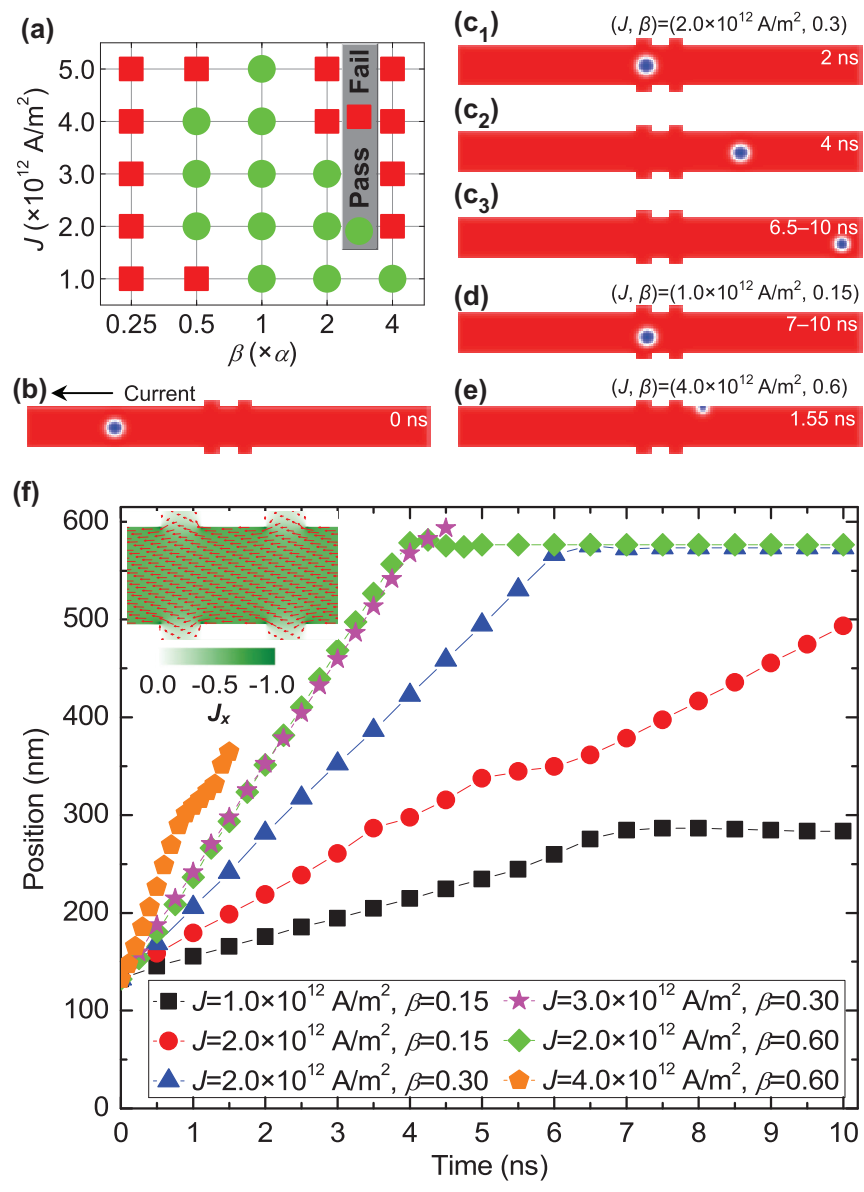

FIG. 3. Current-driven skyrmion motion. (a) Skyrmion behavior versus driving parameters. "Pass" means that the skyrmion can pass through the junction and reach the right terminal of the nanotrack; "Fail" denotes that the skyrmion cannot pass through the junction. Note that such a plot is a working window [33] for current-induced skyrmion-DW pair motion and is not a phase diagram because the latter requires finite compact regions in the phase space $[48,55,75]$. (b) Initial magnetization state at $0 \mathrm{~ns}$ when the current is switched on. (c-e) Skyrmion configuration at special time after current turn-on. (f) Skyrmion position against current-action time for individual $(J, \beta)$ combinations. Zero nanometer and $600 \mathrm{~nm}$ correspond to the left and right terminals of the nanotrack, respectively. Inserted is the real current distribution in the nanotrack, indicating the inhomogeneity in the current flow around the antinotches. The color bar codes $J_{x}$, the $x$ component of the normalized current density, and the red arrows represent the in-plane component.

the resulting collision dynamics of the skyrmion and DW, with several $\beta / \alpha$ values being considered in simulations to account for the divergent results about $\beta$ reported experimentally [50-53].

We deliver the dynamics of a single skyrmion (Fig. 3) and a single DW pair (Fig. 4) in addition to the collision dynamics of the skyrmion and DW pair (Figs. 5-7) with the current being applied along the nanotrack. It is seen that, in a certain range of $J$, the single skyrmion moves smoothly along the track and can freely pass through the junction, whereas the single DW pair, albeit slightly distorted, remains trapped inside the junction, and for the coexisting skyrmion and DW 


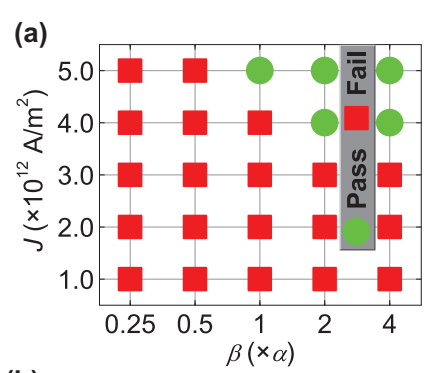

(b)
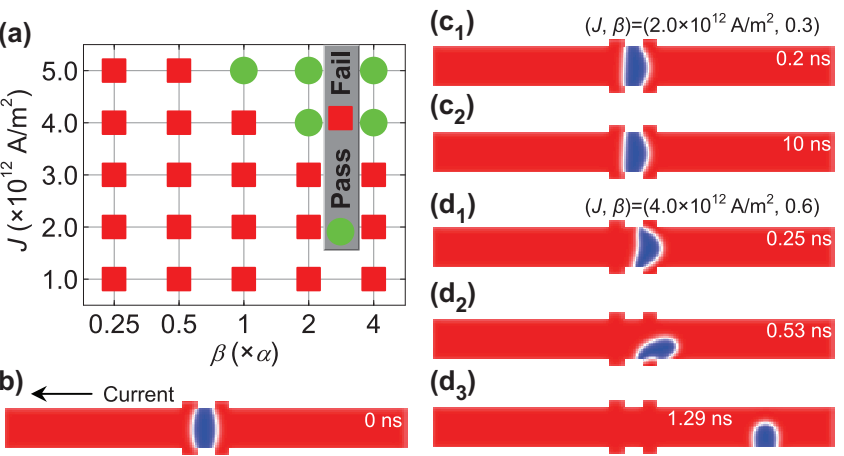

$\left(d_{3}\right)$

(e)

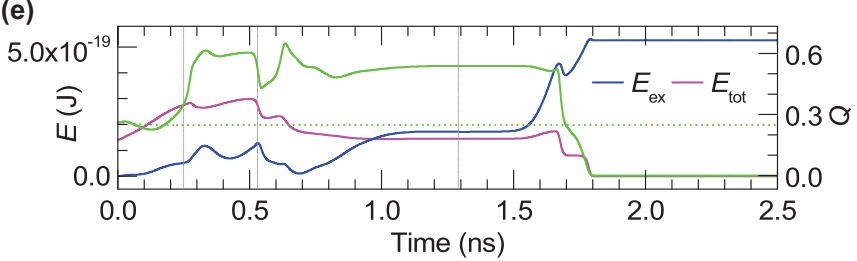

FIG. 4. Current-driven DW pair dynamics. (a) DW pair behavior versus driving parameters. Pass (Fail) means that the DW pair can (cannot) depin from the junction and reach the right terminal. (b) Initial magnetization state at $0 \mathrm{~ns}$ when the current is switched on. (c,d) Spin configuration at special time after current turn-on. In (d), the DW pair actually converts into an edge meron. (e) Energies $E_{\mathrm{ex}}$, $E_{\text {tot }}$, and topological charge $Q$ against current-action time. The solid lines are for (d). The dashed line for (c), displaying $Q$ against time, is flat, in agreement with the tiny structural change in the DW pair for $(J, \beta)=\left(2.0 \times 10^{12} \mathrm{~A} \mathrm{~m}^{-2}, 0.3\right)$.

pair system, the skyrmion can move towards and then collide with the DW pair. By simply varying $J$, we find a series of comotion modes for the colliding skyrmion and DW pair. Especially intriguing is a motion mode triggered by some relatively low current densities, under which the skyrmion firstly propagates toward and eventually is stopped ahead of the junction by the pinned DW pair. Using this character, we build a skyrmionic logic NOT gate and construct logical NAND and NOR gates by assembling two NOT gates in parallel and in series, respectively (Fig. 8). Complex circuits, capable of performing arbitrary logic operations, are expected to be implemented by combining these basic components (i.e., NOT, NAND, and NOR gates). This DW-gated skyrmion logic architecture, together with racetrack memory [28,29], might open opportunities towards skyrmion-based computation.

\section{MODEL AND METHOD}

The nanotrack is a laterally confined ultrathin multilayer film [Fig. 1(a)] with asymmetric interfaces to engender an interfacial DMI [8]. A skyrmion and a DW pair [Fig. 1(b)] can be written into the nanotrack using perpendicular currents flowing through two separate nanocontact spin valves at the positions denoted by the yellow elements [Fig. 1(a)]. The $\mathrm{H}$-shaped junction is used mainly to stabilize the DW pair immersed in an in-plane current, although it can also assist the nucleation of the DW pair induced by a perpendicular current. In the following, we will describe, firstly, the injection process of a DW pair under a vertical current (Fig. 2). The injection of a skyrmion with a perpendicular current is not covered here, because it has already been numerically delivered in Refs. [19,30,54,55]. Subsequently, we will examine successively the motion dynamics of a skyrmion, a DW pair, and the coexisting skyrmion and DW pair triggered by an in-plane current. For these studies, the nanotrack used is $600 \mathrm{~nm}$ long and $60 \mathrm{~nm}$ wide [see Fig. 1(b) for the definition of geometric parameters]. Finally, we will demonstrate the logic NAND and NOR operations. To this end, we assemble single nanotracks to form composite structures. For all simulations, the nanotracks are $1 \mathrm{~nm}$ in thickness.

Micromagnetic simulations, using the public-domain GPU code, MUMAX3 [56], were carried out to unravel the dynamics of the skyrmion and/or the DW pair under electric currents by numerically solving the Landau-Lifshitz-Gilbert (LLG) equation [57,58] augmented with a spin-torque term, which is either in the Slonczewski form [59] or the Zhang-Li form [60] depending on the current direction (perpendicular or in plane). For all computations, the interfacial DMI term was incorporated into the conventional LLG equation [41]. The simulation volume was discretized into an ar-
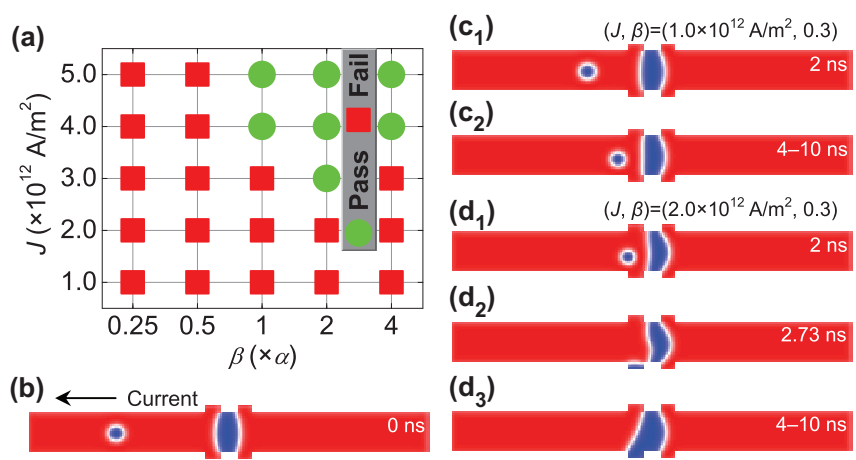

$\left(d_{3}\right)$

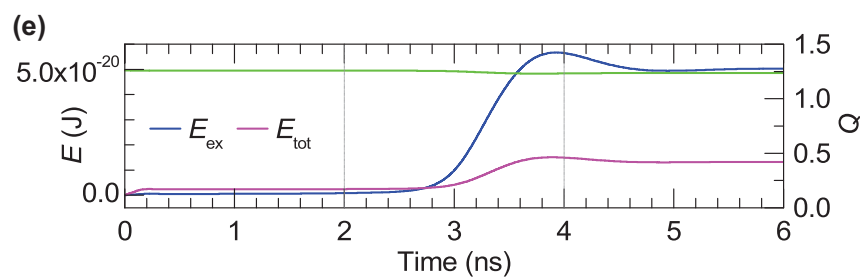

(f)

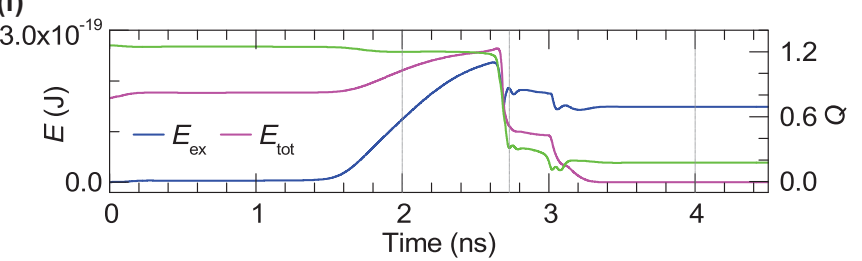

FIG. 5. Current-driven skyrmion DW collision dynamics. (a) Gating effect versus driving parameters. Pass means that one or more magnetic objects resulting from the skyrmion DW collision can pass the junction and reach the right terminal; Fail denotes that no magnetic object can pass through the junction and reach the right terminal. (b) Initial magnetization state at $0 \mathrm{~ns}$ when the current is switched on. (c,d) Spin configuration at special time after current turn-on. In (c), the skyrmion is stopped ahead of the DW pair; in (d), the skyrmion is expelled from the lower boundary. (e,f) Energies $E_{\mathrm{ex}}$, $E_{\text {tot }}$, and topological charge $Q$ against current-action time. Panels $(\mathrm{e}, \mathrm{f})$ correspond to $(\mathrm{c}, \mathrm{d})$, respectively. 
ray of regular meshes $1 \times 1 \times 1 \mathrm{~nm}^{3}$ for finite-difference computation. Material parameters used in simulations are typical of the $\mathrm{Co} / \mathrm{Pt}$ multilayer system with perpendicular magnetic anisotropy (PMA) [3,19,61]: the saturation magnetization $M_{\mathrm{s}}=580 \mathrm{kA} \mathrm{m}^{-1}$, the exchange stiffness $A=15 \mathrm{pJ} \mathrm{m}^{-1}$, the damping constant $\alpha=0.3$, and the electron spin polarization $P=0.4$. For the in-plane current, five representative cases of $\beta=0.25 \alpha, 0.5 \alpha, \alpha, 2 \alpha$, and $4 \alpha$ were considered, where $\beta$ stands for the nonadiabaticity efficiency of the Zhang-Li torque. A series of $K_{\mathrm{u}}$ (perpendicular magnetocrystalline anisotropy) and $D$ (DMI strength) combinations were checked in computations to ensure that the obtained results could be applied to a variety of samples [5,42,44,62]. The collision outcomes for various $K_{\mathrm{u}}$ and $D$ were similar qualitatively. We examined the effect of current distribution on the collision dynamics and did not see an obvious difference for assumed uniform current density and realistic current distribution including current inhomogeneity around antinotches [see inset in Fig. 3(f)]. We also used a commercial code (LLG micromagnetic simulator [63]) to test the reproducibility of the results generated by using MUMAX3 and did not see a significant difference in the collision dynamics [except an upshift of the current density values given in the working windows; see Figs. 3(a), 4(a), and 5(a)]. Therefore, we believe that the effect of domain wall-gated skyrmion motion is robust against varying parameters. The presented results correspond to $K_{\mathrm{u}}=0.8 \mathrm{MJ} \mathrm{m}^{-3}$ [the effective uniaxial anisotropy $K_{\text {eff }}=$ $0.6 \mathrm{MJ} \mathrm{m}^{-3}$ according to $\left.K_{\mathrm{eff}}=K_{\mathrm{u}}-(1 / 2) \mu_{0} M_{\mathrm{s}}^{2}\right]$ and $D=$ $3.5 \mathrm{~mJ} \mathrm{~m}^{-2}$.

\section{RESULTS}

\section{A. Injection of a confined domain wall pair}

Figure 2(a) shows the side view (seen from the $y$ axis) of the nanocontact used to polarize the perpendicular current and shape its path. The current flows across a local area [as enclosed by the yellow box in Fig. 2(b)] in the Co layer beneath the spacer layer, wherein the magnetic moments sense a spin torque. The injection process of the DW pair is illustrated in Fig. 2(c). After current switch-on, the magnetic moments at the edges reverse at first forming two edge merons [64] [Fig. $2\left(c_{1}\right)$ ], as revealed by the increasing topological charge [Fig. 2(d)]. Next, the edge merons expand in size [Fig. 2( $\left.c_{2}\right)$ ] and approach each other generating an antivortex [65] in between [Fig. 2(c $\left.c_{3}\right)$, when the total energy of the system reaches a peak [Fig. 2(e)]. Further, the two distorted edge merons touch each other [Fig. 2( $\left.\mathrm{c}_{4}\right)$ ]—with a quasi-Bloch-point [66] nucleated in between and rapidly annihilated - before merging into a DW pair [Fig. 2( $\left.\mathrm{c}_{5}\right)$ ]. In the $20 \mathrm{ps}$ time from 170 to $190 \mathrm{ps}$, the exchange energy, total energy, and topological charge are dissipated through releasing the Bloch-point-like entity [Fig. 2(e)]. Finally, a stable DW pair is formed inside the junction, as indicated by the unchanging magnetization, topological charge, and energy curves in Figs. 2(d) and 2(e).

The applied current must be sufficiently large to overcome the energy barrier associated with the merging of the two edge merons that repel each other, and it must be reasonably small to prevent the final DW pair from extending outside the junction by suppressing rapid rotation of the magnetic moments directly under the current as well as their strong correlation with external magnetic moments. The edge magnetic moments respond firstly because they have in-plane components (due to the DMI) and thus the angular-momentum transfer between these magnetic moments and the spin-polarized electrons is more efficient than that between the center magnetic moments and the electron spins.

\section{B. Current-driven motion of a single skyrmion}

The spin valve for skyrmion injection is analogous in structure as for DW injection except for the difference in lateral geometry; the detailed skyrmion-injection process has been presented in the literature [19,30,54] and thus is not addressed here. In simulations, we preset a bubblelike spin configuration [67] and relax it to obtain the equilibrium skyrmion for dynamics study. Figure 3(a) plots the skyrmion motion modes evolving with the current density $J$ and the nonadiabaticity coefficient $\beta$. Depending on the values of $J$ and $\beta$, the skyrmion can pass through the junction [denoted as "Pass" in Fig. 3(a)], be blocked by the junction [denoted as "Fail" in Fig. 3(a)], or be ejected from the side boundary [also denoted as Fail in Fig. 3(a)], as shown sequentially in Figs. 3(c)-3(e). Note that Pass requires that the magnetic object, after passing the junction area, can reach the right terminal of the nanotrack; Fail denotes the opposite situation.

The reasons why the skyrmion is stopped at the junction for $(J, \beta)=\left(1.0 \times 10^{12} \mathrm{~A} \mathrm{~m}^{-2}, 0.15\right)$ are firstly, the junction's inclusion modifies the energy landscape [46] of the nanotrack and introduces local potential wells around the antinotches, and secondly, the skyrmion moves toward the side boundary as a consequence of skyrmion Hall effect [24,68] (i.e., a skyrmion moves transversely because of the Magnus force $\left[F_{g} \propto(1-\beta / \alpha) J\right]$ associated with its longitudinal motion $[19,45])$ and drops into the potential well and cannot escape from it because of the small spin torques [adiabatic term $\tau_{\mathrm{a}} \propto J$ and nonadiabatic term $\tau_{\mathrm{b}} \propto \beta J$ [60]; therefore both are the smallest among the cases considered in Fig. 3(a)]. By contrast, skyrmion expulsion at $(J, \beta)=\left(4.0 \times 10^{12} \mathrm{~A} \mathrm{~m}^{-2}, 0.6\right)$ is simply caused by the skyrmion Hall effect and is unrelated to the junction's presence. Notice that for $\beta=(1 / 4) \alpha$ and $4 \alpha$, the skyrmion can hardly penetrate the junction and reach the right terminal regardless of the current density used. For such $(J, \beta)$ values resulting in a large Magnus force, a skyrmion would be annihilated at the side boundary even without including the junction.

The skyrmion position versus time curves [Fig. 3(f)] can provide more information about the skyrmion motion dependent on $(J, \beta)$. The skyrmion under $(J, \beta)=(1.0 \times$ $\left.10^{12} \mathrm{~A} \mathrm{~m}^{-2}, 0.15\right)$ moves initially at a constant speed and stops eventually when it reaches the junction, as revealed in Fig. 3(d). Once falling into the potential well, the skyrmion is tightly bound there unless a stronger current is applied. The difference between the skyrmion motions under $(J, \beta)=$ $\left(2.0 \times 10^{12} \mathrm{~A} \mathrm{~m}^{-2}, 0.15\right)$ and $\left(1.0 \times 10^{12} \mathrm{~A} \mathrm{~m}^{-2}, 0.15\right)$ is that, for the former $(J, \beta)$, the skyrmion can escape from the potential well, because the current's driving force exceeds the well's restoring force. For $(J, \beta)=\left(2.0 \times 10^{12} \mathrm{~A} \mathrm{~m}^{-2}, 0.3\right)$, no Magnus force acts on the skyrmion [because $F_{g} \propto(1-$ $\beta / \alpha) J=0$ for $\beta=\alpha$ ], so that the skyrmion moves along the 
middle axis of the nanotrack when it is far from the right end [Fig. 3(c)], resulting in a constant slope in the position versus time curve [Fig. 3(f)]. The situation for $(J, \beta)=(3.0 \times$ $\left.10^{12} \mathrm{~A} \mathrm{~m}^{-2}, 0.3\right)$ is the same as for $\left(2.0 \times 10^{12} \mathrm{~A} \mathrm{~m}^{-2}, 0.3\right)$ except for the increased skyrmion velocity. The skyrmion ejection at $(J, \beta)=\left(4.0 \times 10^{12} \mathrm{~A} \mathrm{~m}^{-2}, 0.6\right)$ manifests itself as vanishing data points in the position versus time curve after $1.55 \mathrm{~ns}$. We also note that the skyrmion slows down after it enters the junction region and recovers to its initial speed once it leaves the junction, which can be attributed to the dragging of the potential well. When $J$ is decreased to $2.0 \times 10^{12} \mathrm{~A} \mathrm{~m}^{-2}$ ( $\beta$ kept at 0.6), the skyrmion sees negligible influence of the junction, as revealed by the constant slope of the position versus time curve. In this case, the skyrmion's trajectory slightly deviates from the middle axis of the nanotrack because of a tiny Magnus force [recall that $F_{\mathrm{g}} \propto(1-\beta / \alpha) J[19,45]$; here, $J$ is relatively small despite $1-\beta / \alpha \neq 0$ ].

Based on Fig. 3, we can conclude that the skyrmion motion behaviors in the junction-contained nanotrack are much similar as in a homogeneous nanotrack for most cases except $(J, \beta)=\left(1.0 \times 10^{12} \mathrm{~A} \mathrm{~m}^{-2}, 0.5 \alpha\right)$ and $(1.0 \times$ $\left.10^{12} \mathrm{~A} \mathrm{~m}^{-2}, 0.25 \alpha\right)$. That is to say, inclusion of the junction does not significantly change the current-induced skyrmion motion behaviors.

\section{Current-driven dynamics of a confined domain wall pair}

Figure 4 presents the results for current-driven dynamics of a confined DW pair. As seen from Fig. 4(a), for most $(J, \beta)$ combinations, the DWs fail to escape from the confining potential of the junction. Among the considered cases, only for the largest $(J, \beta)$ can the DW pair succeed in passing through the junction. Figure 4(c) displaying the DW pair under $(J, \beta)=$ $\left(2.0 \times 10^{12} \mathrm{~A} \mathrm{~m}^{-2}, 0.3\right)$ indicates that the profile of the DW pair is slightly modified immediately after current application [e.g., 0.2 ns; Fig. 4( $\left.c_{1}\right)$ ], and no more variation occurs to the structure from 0.2 to $10 \mathrm{~ns}$ [compare Figs. $4\left(\mathrm{c}_{1}\right)$ and $4\left(c_{2}\right)$ ], suggesting that the DW pair cannot be released by such small driving forces even for a long action time. For $(J, \beta)=\left(4.0 \times 10^{12} \mathrm{~A} \mathrm{~m}^{-2}, 0.6\right)$, the DW pair is firstly bent and then twisted [Fig. $4\left(\mathrm{~d}_{1}\right)$ ], resulting in an asymmetric DW profile with respect to the nanotrack's middle axis, which might be attributed to the DMI $[29,42,43]$. Subsequently, the near and far DWs coalesce at around the upper edge, where they meet because of heavy distortion. In this way, a DW pair, with two open ends pinned at opposite edges, is depinned from one edge, resulting in an intermediate magnetic entity with one open end, as shown in Fig. $4\left(\mathrm{~d}_{2}\right)$. After escaping from the junction, the magnetic object converts into an edge meron [64], which moves steadily along the boundary and ultimately leaves the nanotrack at the right terminal. The complex evolution process from the DW pair to the edge meron manifests itself as the changing topological charge and energies of the system [Fig. 4(e)].

By comparing Figs. 3(a) and 4(a), one finds that for most $(J, \beta)$ combinations-under which the single skyrmion can pass the junction-the DWs are tightly trapped inside the junction. Namely, the junction can effectively pin the DWs and hardly affect the skyrmion motion for $\beta$ not far different from $\alpha$.

\section{Current-driven collision dynamics of skyrmion and domain wall pair}

Now, we turn to the collision dynamics of the coexisting skyrmion and DW pair in response to an in-plane current. Whether the trapped DWs can stop the original skyrmion (or other secondary entities) relies on the driving parameters, i.e., $(J, \beta)$, as shown in Fig. 5(a). One can see that for lower values of $(J, \beta)$, no magnetic objects can penetrate the junction. Only when $J$ and $\beta$ both become sufficiently large can some magnetic objects enter and move steadily along the right branch of the nanotrack. Next, we analyze the collision processes of the skyrmion and DWs in detail.

For $(J, \beta)=\left(1.0 \times 10^{12} \mathrm{~A} \mathrm{~m}^{-2}, 0.3\right)$, the skyrmion is $\mathrm{fi}-$ nally blocked in front of the DW pair, as shown in Fig. 5(c). Here, the current-induced force cannot overcome the repulsion force from the near DW as the skyrmion moves to it, and eventually the two forces balance out at a certain distance between the skyrmion and the near DW. Increasing $J$ while keeping $\beta$ unchanged will lead the skyrmion to be expelled from the boundary [Fig. 5(d)]. The detailed process is as follows: As the skyrmion approaches the near DW, the repulsion force between them further distorts the DW pair, so that the asymmetry $[42,43]$ of the DW pair relative to the long axis of the nanotrack is enhanced and in turn leads to a sizable transverse (i.e., along the $y$ axis) force on the skyrmion. As a result, the skyrmion moves transversely [Fig. $5\left(\mathrm{~d}_{1}\right)$ ], touches the lower-left antinotch of the junction [Fig. $5\left(\mathrm{~d}_{2}\right)$ ], and finally is ejected from the antinotch. Under the repulsion force of the far DW, the near DW moves backward and finally extends beyond the junction [Fig. $5\left(\mathrm{~d}_{3}\right)$ ]. However, the entire DW pair is still pinned at the junction.

To further understand the dynamic processes in Figs. 5(c) and 5(d), we plot $Q(t)$ and $E(t)$ curves in Figs. 5(e) and 5(f). From Fig. 5(e), we see that $Q$ changes only slightly with time, which is because no drastic structural variation happens to the skyrmion or DW pair during the current action. The current serves simply to move the skyrmion towards the DW pair. The jump in exchange energy before 4 ns corresponds to the increasing repulsion between the skyrmion and near DW. Nevertheless, from Fig. 5(f), we see a sharp decrease in $Q$ and $E$ at $\sim 2.73$ ns [Fig. $5\left(\mathrm{~d}_{2}\right)$ ], which characterizes the breakdown of the skyrmion texture at the boundary [45].

Further increasing the current density while maintaining $\beta$ at 0.3 will cause the DW pair to partially detach from the junction. The whole process is depicted in Fig. 6(a), where the initial process before $1.6 \mathrm{~ns}$ is similar to that before $2.7 \mathrm{~ns}$ for $(J, \beta)=\left(2.0 \times 10^{12} \mathrm{~A} \mathrm{~m}^{-2}, 0.3\right)$; compare Fig. 6(c) with Fig. 5(f). At this stage, the skyrmion approaches the DW pair causing enhanced mutual repulsion; consequently, the DW pair distorts and the skyrmion shrinks in size, deviates from the nanotrack's middle axis, and ultimately touches the boundary becoming a fractional skyrmion [29]. After $1.6 \mathrm{~ns}$, the fractional skyrmion continues to diminish, and remarkably, the DW pair, enclosed by two open strings, is depinned from the lower edge, becoming the other fractional skyrmion encircled by a single string [Fig. 6(a $\left.\mathrm{a}_{5}\right)$ ], for which $Q$ is $\sim 0.65$ [Fig. 6(c)]. Under the current action, this fractional skyrmion reaches a dynamic equilibrium configuration, which is maintained by three independent forces, i.e., $\mathbf{F}_{\mathrm{v}}, \mathbf{F}_{\mathrm{g}}$, and 


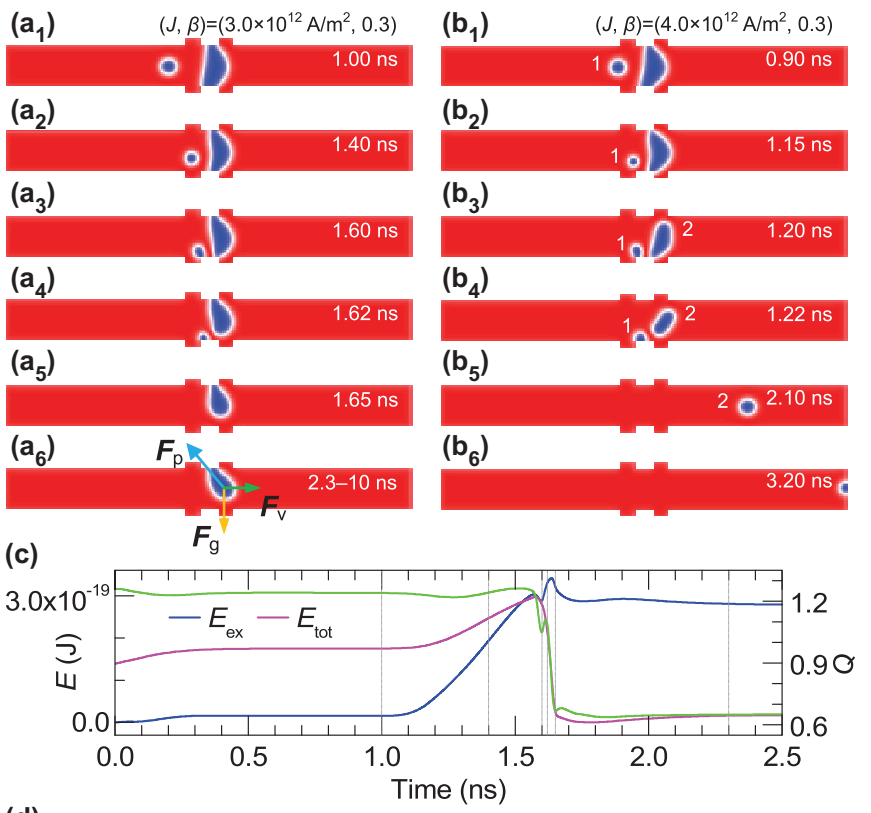

(d)

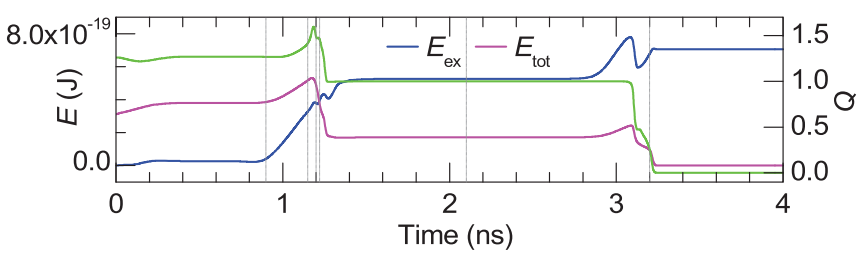

FIG. 6. Skyrmion DW collision at increased current densities with $\beta=\alpha$. (a,b) Spin configuration at special time after current turn-on. In both $(a, b)$, the original skyrmion is ejected from the lower boundary. In (a), the DW pair is partially depinned from the junction, becoming a dynamically stabilized fractional skyrmion; in (b), the DW pair is converted into a new skyrmion. (c,d) Energies $E_{\mathrm{ex}}, E_{\mathrm{tot}}$, and topological charge $Q$ against current-action time. Panels (c,d) correspond to $(\mathrm{a}, \mathrm{b})$, respectively.

$\mathbf{F}_{\mathrm{p}}$, as shown in Fig. 6(a $\left.\mathrm{a}_{6}\right)$. Once the current is switched off, the fractional skyrmion decays rapidly and is ejected from the boundary after $\sim 0.6 \mathrm{~ns}$ (see Fig. S1 in the Supplemental Material [69] for details), since the force balance between $\mathbf{F}_{\mathrm{v}}$, $\mathbf{F}_{\mathrm{g}}$, and $\mathbf{F}_{\mathrm{p}}$ is broken. On the other hand, if the current density increases to $4.0 \times 10^{12} \mathrm{~A} \mathrm{~m}^{-2}$, the fractional skyrmion will be pulled into the nanotrack becoming a skyrmion (see Fig. S2 [69]).

Force analysis. The forces-experienced by the fractional skyrmion in equilibrium-should satisfy the Thiele equation that reads $[17,19,39,45,70]$

$$
\mathbf{G} \times\left(\mathbf{v}^{\mathrm{s}}-\mathbf{v}^{\mathrm{d}}\right)-\nabla V+\mathcal{D}\left(\beta \mathbf{v}^{\mathrm{s}}-\alpha \mathbf{v}^{\mathrm{d}}\right)=0,
$$

which describes the balance of the Magnus force $\left[\mathbf{F}_{\mathrm{g}}=\mathbf{G} \times\right.$ $\left.\left(\mathbf{v}^{\mathrm{s}}-\mathbf{v}^{\mathrm{d}}\right)\right]$, the confining force $\left(\mathbf{F}_{\mathrm{p}}=-\nabla V\right)$, and the viscous force $\left[\mathbf{F}_{\mathrm{v}}=\mathcal{D}\left(\beta \mathbf{v}^{\mathrm{s}}-\alpha \mathbf{v}^{\mathrm{d}}\right)\right] . \mathbf{G}=G \hat{\mathrm{e}}_{z}$ is a gyrocoupling vector with $G$ being proportional to $Q$ and $\hat{\mathrm{e}}_{z}$ representing the unit vector along the $z$ axis, $V$ is the confining potential due to boundaries, and $\mathcal{D}=\left(\begin{array}{ll}\mathcal{D}_{x x} & \mathcal{D}_{x y} \\ \mathcal{D}_{y x} & \mathcal{D}_{y y}\end{array}\right)=\left(\begin{array}{ll}\mathcal{D} & 0 \\ 0 & \mathcal{D}\end{array}\right)$ is a dissipation tensor. $\mathbf{v}^{\mathrm{d}}$ is the drift velocity of a spin texture, and $\mathbf{v}^{\mathrm{s}}=$ $-\left[\gamma \hbar P /\left(2 \mu_{0} e M_{s}\right)\right] J \hat{e}_{x}$ is the velocity of conduction electrons
[60], where $\hat{\mathrm{e}}_{x}$ is the unit vector along $x ; \gamma$ the gyromagnetic ratio; $\mu_{0}$ the vacuum permeability; $\hbar$ the reduced Planck constant; and $e$ the elementary charge. Here, the fractional skyrmion is static (actually, dynamically stabilized), i.e., $\mathbf{v}^{\mathrm{d}}=0$; therefore, $\mathbf{F}_{\mathrm{g}}=\mathbf{G} \times \mathbf{v}^{\mathrm{s}}$ and $\mathbf{F}_{\mathrm{v}}=\mathcal{D} \beta \mathbf{v}^{\mathrm{s}}$, which allows one to identify the directions of the two forces [as labeled in Fig. 6(a $\left.a_{6}\right)$ ]. In the present case, the confining force $\mathbf{F}_{\mathrm{p}}$ arises sorely from the boundaries and acts as a passive force to counteract against the Magnus and viscous forces, leading to zero net force upon the fractional skyrmion.

If the current density increases further such that $(J, \beta)=$ $\left(4.0 \times 10^{12} \mathrm{~A} \mathrm{~m}^{-2}, 0.3\right)$, the DW pair will convert into a skyrmion, as illustrated in Fig. 6(b). The process of the first $1 \mathrm{~ns}$ still resembles the initial processes described above [compare Figs. 6(a $\left.a_{2}\right)$ and $6\left(b_{2}\right)$ ]. Because of the increased current density, the skyrmion is closer to the DW pair, making the latter undergo stronger distortion. As a result, the two DWs merge at the upper boundary forming a fractional skyrmion [2 in Fig. 6(b $\left.b_{3}\right)$ ], different from what occurs in Fig. 6(a), and meanwhile, the skyrmion touches the lower boundary giving the other fractional skyrmion (1). Next, as the fractional skyrmion 1 continues to move rightward, the fractional skyrmion 2 is forced to depin from the junction and transform into an elongated magnetic bubble [19,29] [Fig. 6( $\left(b_{4}\right)$ ]. Subsequently, the fractional skyrmion 1 leaves the nanotrack and the bubble (2) relaxes into a regular skyrmion. Thereafter, the new skyrmion moves along the nanotrack and is annihilated at the right terminal. The two events, i.e., conversion from a DW pair to a skyrmion and annihilation of the new skyrmion, manifest themselves as two abrupt drops in the $Q(t)$ curve in Fig. 6(d), from $\sim 1.5$ to 1.0 and 1.0 to 0 , respectively.

In Figs. 5 and 6, the dynamic processes for $\beta=0.3$ are presented in detail. Now, we go into the details for $\beta=0.6$ (Fig. 7). For $(J, \beta)=\left(3.0 \times 10^{12} \mathrm{~A} \mathrm{~m}^{-2}, 0.6\right)$, because of a Magnus force, the skyrmion moves upward $[19,45]$ when it approaches the DW pair [Fig. 7( $\left.\mathrm{a}_{1}\right)$ ]. The DW pair depins from the junction at the upper boundary [Fig. 7( $\left.\mathrm{a}_{2}\right)$ ] in response to the repulsion force from the skyrmion. The newly generated fractional skyrmion no longer detaches from the lower boundary as in Figs. 6(a) and 6(b), where the repulsion force from the melting skyrmion dominates the depinning of the DW pair. In this case, the compact skyrmion still contributes a repulsion force, which tends to expel the new fractional skyrmion from the nanotrack. Since $\beta \neq \alpha$, a Magnus force happens to the fractional skyrmion and counterbalances the repulsion force from the skyrmion, so that the fractional skyrmion is dynamically protected from being ejected and converts into an edge meron [64] [Fig. 7( $\left.\mathrm{a}_{4}\right)$ ]. After penetrating the junction, the skyrmion and the edge meron move steadily in the right branch and reach the right terminal [Figs. 7( $\left.\left.\mathrm{a}_{5}\right)-7\left(\mathrm{a}_{8}\right)\right]$. The drops in the $Q(t)$ curve in Fig. 7(c) around 1.3, 2.6, and 3.2 ns mark the edge-meron depinning from the junction, the edge-meron annihilation, and the skyrmion annihilation at the right terminal, respectively.

For $(J, \beta)=\left(4.0 \times 10^{12} \mathrm{~A} \mathrm{~m}^{-2}, 0.6\right)$, there is no interaction between the skyrmion and the DW pair because of a long distance between them. Therefore, the motions of the two objects are fully independent. As a result, the motions of the coexisting skyrmion and DW pair [Fig. 7(b)] appear to be a 

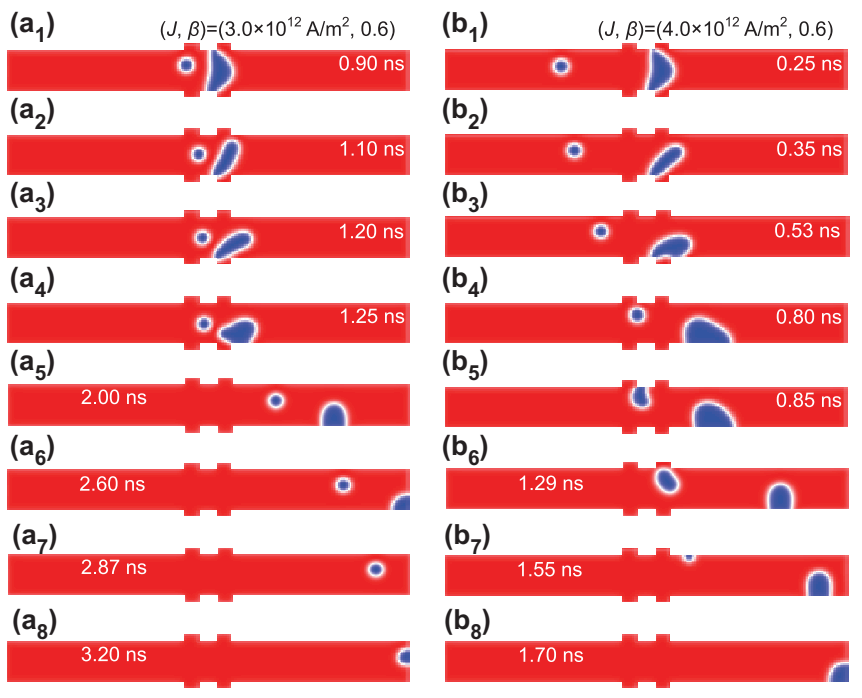

(c)

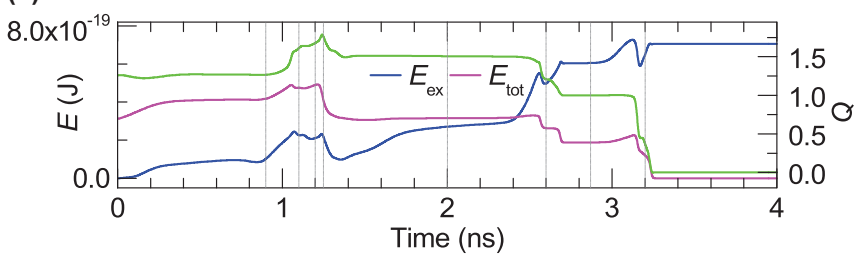

(d)

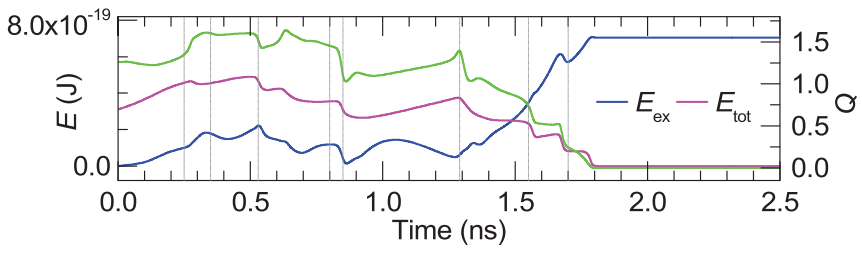

FIG. 7. Skyrmion DW collision under $\beta=2 \alpha$. (a,b) Spin configuration at special time after current turn-on. In both $(a, b)$, the DW pair is converted into an edge meron. In (a), the skyrmion passes through the junction and reaches the right terminal; in (b), the DW pair goes through the junction area but melts immediately at the upper boundary. Note that there is no skyrmion DW interplay in (b); each individual object responds independently to the current-induced torque. (c,d) Energies $E_{\mathrm{ex}}, E_{\mathrm{tot}}$, and topological charge $Q$ against current-action time. Panels (c,d) correspond to (a b), respectively.

superposition of the individual motions of the single skyrmion and the single DW pair (Figs. 3 and 4).

The behaviors of the coexisting skyrmion and DW pair can be briefly summarized as follows. For $(J, \beta)=(1.0 \times$ $\left.10^{12} \mathrm{~A} \mathrm{~m}^{-2}, 0.3\right)$, the skyrmion is blocked in front of the junction by the DW pair, although the latter is slightly modified in shape. For $(J, \beta)=\left(2.0 \times 10^{12} \mathrm{~A} \mathrm{~m}^{-2}, 0.3\right)$, the skyrmion is stopped ahead of the junction and ejected from the lower boundary, and the DW pair is distorted. For $(J, \beta)=\left(3.0 \times 10^{12} \mathrm{~A} \mathrm{~m}^{-2}, 0.3\right)$, the DW pair blocks the skyrmion and pushes it out of the nanotrack, and eventually the DW pair is converted into a fractional skyrmion. For $(J, \beta)=\left(4.0 \times 10^{12} \mathrm{~A} \mathrm{~m}^{-2}, 0.3\right)$, the DW pair blocks the skyrmion by pushing it out of the nanotrack, but meanwhile the DW pair is entirely depinned from the junction and becomes a skyrmion. For $(J, \beta)=\left(3.0 \times 10^{12} \mathrm{~A} \mathrm{~m}^{-2}, 0.6\right)$, the DW pair no longer blocks the skyrmion and is forced to depin from the junction becoming an edge meron. For $(J, \beta)=\left(4.0 \times 10^{12} \mathrm{~A} \mathrm{~m}^{-2}, 0.6\right)$, both the skyrmion and the DW pair overcome the confining potential of the junction and enter the right arm, and afterwards, the skyrmion annihilates immediately at the upper boundary and the DW pair converts into an edge meron.

The rich collision dynamics of the coexisting skyrmion and DW pair embodies the complex competition among the current-induced forces $[19,45]$ (i.e., viscous force and Magnus force), the confining force due to boundaries, and the repulsive force between magnetic objects. Approximately, the higher the current density is, the nearer the skyrmion can approach the DW pair and in turn the stronger the repulsion between the skyrmion and the DW pair. The ejection of the skyrmion from the nanotrack illustrated in Figs. 5(d) and 6 is primarily due to the repulsion force rather than the Magnus force, which, however, is in charge of the skyrmion ejection shown in Fig. 3(e). The strength and direction of the repulsive force are determined by the shapes, sizes, positions, and spacing of the skyrmion and the DW pair, which are rapidly evolving under the simultaneous action of the aforementioned forces. Furthermore, as presented above, the Magnus and viscous forces are functions of the drift velocity $\left(\mathbf{v}^{\mathrm{d}}\right)$ of a magnetic object, and the confining force relies on the shape, size, and position of a magnetic object $[19,45,70]$. Therefore, the forces and the motional states are mutually dependent. Note that there is fundamental difference between the dynamics of a geometrically confined single DW and a DW forming the DW pair driven by the in-plane currents because the pairing DWs interact with each other. Consequently, it is difficult to compare the depinning dynamics of a single DW and the pairing DWs from the $\mathrm{H}$-shaped junction. Here, the skyrmion DW collision further complicates the situation since the approaching skyrmion exerts an additional repulsive force on the DW pair.

\section{E. Logic operations by domain wall-mediated skyrmion motion}

Now, let us neglect the detailed motion processes of the skyrmion and the DW pair and simply concentrate on the gating effect of the DW pair on the skyrmion by comparing Figs. 3(a), 4(a), and 5(a). Clearly, for most $(J, \beta)$ combinations with $\beta$ not far different from $\alpha$, if no DW pair is formed in the junction, a skyrmion can pass through the junction; if a DW pair is present in the junction, a skyrmion cannot pass, since it is either stopped in front of the DW pair or ejected from the nanotrack. This gating effect is indeed equivalent to a logic NOT operation [27], for which the presence of the DW pair (skyrmion) in the junction (right branch) of the nanotrack is encoded into " 1 " at the input (output) terminal and their absence into " 0 ." In this logic NOT gate, the skyrmion behaves as a carrier of information and the DW pair as a gatekeeper that controls the information flow.

A logic NOT gate is a 1-input signal-processing component; nevertheless, before any arbitrary logical functions can be implemented, at least one 2-input gate has to be constructed preliminarily [27]. By virtue of the structural characters of the logic NOT gate, we propose two types of such componentsNAND and NOR gates [71-73]. The schematic architectures for them are shown in Fig. 8. The NAND gate is built from two 


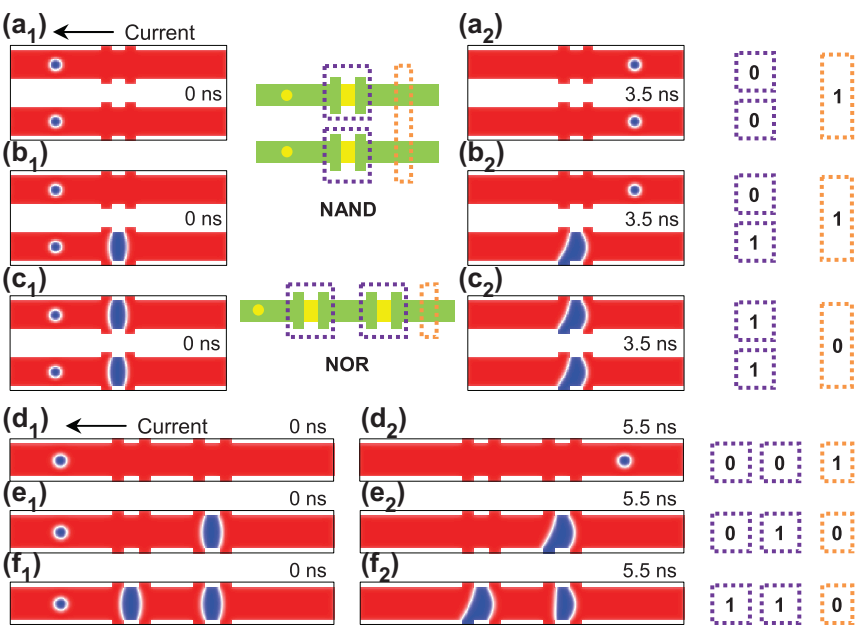

FIG. 8. Layout and operation of logic NAND and NOR functions. Two single NOT gates connected in parallel or in serial make a NAND or a NOR gate. The purple and orange boxes denote the input and output ports, respectively. (a-c) Logic NAND gate. (d-f) Logic NOR gate. The left panels show the initial states before operation, and the right panels give the results after operation. The truth tables are attached on the right. For each operation, a current with $(J, \beta)=$ $\left(2.0 \times 10^{12} \mathrm{~A} \mathrm{~m}^{-2}, \alpha\right)$ is used as a driving force. The current-action time is $3.5 \mathrm{~ns}$ for NAND and $5.5 \mathrm{~ns}$ for NOR.

NOT gates connected in parallel, and the NOR gate is based on two serially connected NOT gates. For each 2 -input gate, the two inputs are encoded into the individual magnetic states of the two junctions, and the output is encoded into the magnetic state of the right arm.

We numerically test the functionality of the proposed 2-input gates using $(J, \beta)=\left(2.0 \times 10^{12} \mathrm{~A} \mathrm{~m}^{-2}, \alpha\right)$, and the operation results for the NAND and NOR gates are illustrated in Figs. 8(a)-8(c) and 8(d)-8(f), respectively. The left panels show the initial states for each operation. A current is switched on at $0 \mathrm{~ns}$ to move the skyrmion carrier, and for the NAND (NOR) gate the magnetic state at the output port is measured at $3.5 \mathrm{~ns}$ ( $5.5 \mathrm{~ns})$. Once one or more skyrmions are detected, the output will be recognized as 1 ; otherwise, as 0 . The detection can be achieved using GMR/TMR effect or by virtue of the emergent electrodynamics intrinsic to magnetic skyrmions. Using this coding scheme, the truth tables for the two gates are derived. Note that the operation details of $1 \mid 0 \rightarrow 1$ for NAND and $1 \mid 0 \rightarrow 0$ for NOR are omitted here, since they resemble those of $0 \mid 1 \rightarrow 1$ and $0 \mid 1 \rightarrow 0$, respectively.

\section{DISCUSSION}

The domain walls (actually, a domain wall pair) in the proposed system have a fixed initial position defined by the $\mathrm{H}$-shaped junction. Moreover, the domain wall pair exhibits simple static structure for the considered magnetic nanotracks with PMA and DMI. The initial position of the skyrmion is determined by the left injection spin valve, which is far away from the right injection spin valve situated atop the junction to ensure the initial skyrmion and DW pair are decoupled with each other. Therefore, the initial positions of the skyrmion and DW pair can be well controlled.
The real-time structures and positions of the skyrmion and DW pair after current switch-on are totally determined by the driving force itself for given initial conditions (initial structures and positions of magnetic objects, material and geometric parameters, and current parameters). Indeed, the collision outcomes depend on the structures and positions of the skyrmion and DW pair, which are in turn determined by the applied driving force, as explained above. Thus, one can reasonably expect that the collision outcomes are fully dependent on the driving force for specific initial conditions, because every transient state of the system after current turn-on evolves from the state of the former moment (neglecting random effects).

The distance between the skyrmion and DW pair does not affect the collision dynamics so long as the skyrmion and the DW pair are not too near, because the individual structures of the skyrmion and the DW pair are determined by the geometry and material parameters of the nanotrack. Only when the skyrmion and the DW pair are close enough to repel each other, their own structures (shapes, sizes, and configurations) are sensitive to their distance. In this study, the initial distance between the skyrmion and DW pair was chosen to be large enough to avoid direct interaction.

Generally, the interactions between domain walls and geometrical restrictions are very complicated, as discussed in Refs. [47-49]. Here, the interaction between the confined domain walls (forming a DW pair inside the $\mathrm{H}$-shaped junction) and the junction antinotches is relatively simple, possibly because of the simple structure of DWs in the system with PMA and DMI; for example, a single DW in our tracks is mirror symmetric about the long axis, as shown in Ref. [74], whereas that in magnetic tracks without PMA is strawberrylike, as shown in Ref. [75].

As a result, the collision outcomes for the DW pair are relatively simple and can be classified into three cases: (i) Both the open ends of the DW pair close and the DW pair converts into a skyrmion, (ii) one of the two open ends of the DW pair coalescences and the DW pair becomes a meron (fractional skyrmion) or a meronlike object, and (iii) neither of the two open ends closes and the DW pair sustains the configuration, i.e., remains as a DW pair.

The presented results should have incorporated as many outcomes of skyrmion domain wall collision as can be enabled by the in-plane currents with the considered $(J, \beta)$ parameters. The results in Figs. 5(c), 5(d), 6(a), 6(b), 7(a), and 7(b) are representative collision outcomes at least for such $(J, \beta)$ combinations where $\beta$ is not much different from $\alpha$ and $J$ is not too high (this parameter range is of great interest for device applications). For a given $(J, \beta)$ satisfying the above condition, the resulting collision dynamics should be described by (at least be analogous to) one of the processes described in Figs. 5(c), 5(d), 6(a), 6(b), 7(a), and 7(b).

Scrutinizing the collision processes, one can find that even for $\beta=\alpha$, the moving skyrmion always deviates from the middle axis once it is close to the near DW. However, in this case no transverse force can arise from a current since $\beta=\alpha$ leads to zero Magnus force $[19,45]$. Therefore, the transverse skyrmion motion should be due to a transverse component of the repulsion force, which can be caused by the asymmetry in the DW profile $[42,43]$. 
Geometrically, a closed string must not be in contact with any boundary; once a string intersects a boundary, it is split apart at the contact point. In other words, if two DW ends merge, the merging point must lie at the boundary. Therefore, in view of string geometry [29], the DW pair after collision can only exist as one of the three configurations, namely, a slightly deformed DW pair, a fractional skyrmion (edge meron), or a skyrmion. In simulations, we indeed observe all three states, which are enabled under various collision conditions determined by the current parameters - $J$ and $\beta$. As to the gating effect of the DW pair, there also exist three outcomes; i.e., the skyrmion is stopped and the DW pair remains confined inside the junction-this situation prevails for most $(J, \beta)$ combinations considered-or the skyrmion is blocked and the DW pair detaches, or the skyrmion passes through the junction and the DW pair is released.

There is repulsion between the two DWs forming the DW pair, especially when the near DW is close to the far DW and/or when it is distorted by a force from either a current or the skyrmion. During collision, the skyrmion is rigid and well preserves its shape (i.e., without distortion) with its size being gradually reduced in approaching the DW. The skyrmion cannot be annihilated unless it intersects a boundary $[38,43]$. On the contrary, the DWs are rather "soft"; they deform in response to the forces exerted by the current and/or the skyrmion. Owing to the softness of the DW pair, there is no restriction on the geometry of the evolving DW pair. As a result, multiple motion modes can be dynamically activated for the coexisting skyrmion and DW pair by varying the driving parameters. Here, the topological property of a spin texture manifests itself again. The skyrmion is protected from being distorted by its quantized topological charge [13,20] regardless of the driving parameters, whereas the DW pair, lacking topological protection, is twisted, stretched, and/or merged depending heavily on the driving parameters.

We would like to mention that Kunz [40] numerically addressed magnetic field-driven collision of transverse DWs in soft magnetic nanowires, which was found to be able to annihilate DWs or render a $360^{\circ} \mathrm{DW}$ depending on the relative alignments of the colliding DWs, and furthermore he demonstrated collision-assisted DW depinning from a notch under an eightfold smaller field. In a seminal work [27], Allwood et al. experimentally demonstrated a set of DW-based logic gates operated using pulsed magnetic fields, which has inspired enormous research activities on both fundamental and technological issues of DWs. In the long term, DWs might have to eventually give way to skyrmions for magnetic memory and logic applications, since the latter promises denser and more-energy-efficient devices [18]. Recently, our group [32] demonstrated another two kinds of skyrmion-based, 2-input logical gates, that is, AND and OR, which are based on a skyrmion-merging architecture and, however, would require sophisticated procedures for practical fabrication. In a later work [33], we proposed a voltage-gated skyrmion transistor, which has a simple structure and can behave as a logical NOT gate, if the input and output are encoded into the control voltage and local magnetization orientation, respectively. Despite the similarity in circuit structure to the voltage-gated gate, the NOT and resulting NAND and NOR gates demonstrated here operate under a unique gating mechanism that benefits from the skyrmion DW interplay.

Magnonics [76] (i.e., magnon spintronics), as a rapidly growing research field, represents another technological route for circumventing the bottleneck of today's CMOS-based technologies. The prominent advantages that magnonic circuitry can offer include the substantially enhanced throughput and energy efficiency resulting from the inherent high group velocity and low power of spin waves $[77,78]$. In this aspect, it is difficult for skyrmion-based devices to outperform spin wave-based ones unless ultrahigh skyrmion mobility can be achieved, for example, by improving spin transfer efficiency between driving currents and local magnetic moments. Compared to spin-wave logic [71-73], skyrmion logic is more compatible with racetrack memory $[18,26,28]$ and thus makes hardware reconfiguration easier, because the former uses magnons rather than skyrmions as information carriers.

It is worth noting that in this proof-of-principle demonstration of DW-gated skyrmion logic, the Zhang-Li form of spin torques is used to trigger skyrmion movement. However, in practical implementation, the device structure and materials can be optimized to benefit from the high efficiency of emerging spin-orbit torques [79-81], promising energy-efficient operations at far reduced current densities. This study helps uncover the difference in the dynamics of topological and topologically trivial magnetic objects, which are inaccessible by simply using external stimuli such as electric currents.

\section{CONCLUSION}

In conclusion, we numerically realize current-driven skyrmion DW collision by introducing a planar junction into a magnetic racetrack, which can selectively trap a DW pair and allows a skyrmion to freely pass through. Using this structure, we systematically study the collision dynamics of coexisting skyrmion and DW pair and identify the conditions for DWgated skyrmion motion. Finally, we propose the concept of DW-gated skyrmion logic and numerically demonstrate a set of elementary logic gates.

\section{ACKNOWLEDGMENTS}

Y.Z. acknowledges the support by National Natural Science Foundation of China (Project No. 1157040329), the Seed Funding Program for Basic Research and Seed Funding Program for Applied Research from the HKU, ITF Tier 3 funding (ITS/203/14), the RGC-GRF under Grant No. HKU 17210014, and University Grants Committee of Hong Kong (Contract No. AoE/P-04/08). X.J.X. acknowledges the support by the Zhejiang Provincial Natural Science Foundation of China under Grant No. LY14A040006 and the National Natural Science Foundation of China under Grant No. 11104206.
[1] S. Mühlbauer, B. Binz, F. Jonietz, C. Pfleiderer, A. Rosch, A. Neubauer, R. Georgii, and P. Böni, Science 323, 915 (2009).
[2] X. Z. Yu, Y. Onose, N. Kanazawa, J. H. Park, J. H. Han, Y. Matsui, N. Nagaosa, and Y. Tokura, Nature 465, 901 (2010). 
[3] S. Heinze, K. von Bergmann, M. Menzel, J. Brede, A. Kubetzka, R. Wiesendanger, G. Bihlmayer, and S. Blugel, Nat. Phys. 7, 713 (2011).

[4] M. Heide, G. Bihlmayer, and S. Blügel, Phys. Rev. B 78, 140403 (2008).

[5] G. Chen, J. Zhu, A. Quesada, J. Li, A. T. N'Diaye, Y. Huo, T. P. Ma, Y. Chen, H. Y. Kwon, C. Won, Z. Q. Qiu, A. K. Schmid, and Y. Z. Wu, Phys. Rev. Lett. 110, 177204 (2013).

[6] I. E. Dzyaloshinskii, Sov. Phys. JETP 5, 1259 (1957).

[7] T. Moriya, Phys. Rev. 120, 91 (1960).

[8] A. Fert, Mater. Sci. Forum 59-60, 439 (1990).

[9] A. Fert and P. M. Levy, Phys. Rev. Lett. 44, 1538 (1980).

[10] T. H. R. Skyrme, Nucl. Phys. 31, 556 (1962).

[11] D. C. Wright and N. D. Mermin, Rev. Mod. Phys. 61, 385 (1989).

[12] U. Al'Khawaja and H. T. C. Stoof, Nature 411, 918 (2001).

[13] N. Nagaosa and Y. Tokura, Nat. Nanotechnol. 8, 899 (2013).

[14] F. Jonietz, S. Muhlbauer, C. Pfleiderer, A. Neubauer, W. Munzer, A. Bauer, T. Adams, R. Georgii, P. Boni, R. A. Duine, K. Everschor, M. Garst, and A. Rosch, Science 330, 1648 (2010).

[15] T. Schulz, R. Ritz, A. Bauer, M. Halder, M. Wagner, C. Franz, C. Pfleiderer, K. Everschor, M. Garst, and A. Rosch, Nat. Phys. 8, 301 (2012).

[16] X. Z. Yu, N. Kanazawa, W. Z. Zhang, T. Nagai, T. Hara, K. Kimoto, Y. Matsui, Y. Onose, and Y. Tokura, Nat. Commun. 3, 988 (2012).

[17] J. Iwasaki, M. Mochizuki, and N. Nagaosa, Nat. Commun. 4, 1463 (2013).

[18] A. Fert, V. Cros, and J. Sampaio, Nat. Nanotechnol. 8, 152 (2013).

[19] J. Sampaio, V. Cros, S. Rohart, A. Thiaville, and A. Fert, Nat. Nanotechnol. 8, 839 (2013).

[20] A. Rosch, Nat. Nanotechnol. 8, 160 (2013).

[21] Y. Nii, T. Nakajima, A. Kikkawa, Y. Yamasaki, K. Ohishi, J. Suzuki, Y. Taguchi, T. Arima, Y. Tokura, and Y. Iwasa, Nat. Commun. 6, 8539 (2015).

[22] A. Neubauer, C. Pfleiderer, B. Binz, A. Rosch, R. Ritz, P. G. Niklowitz, and P. Böni, Phys. Rev. Lett. 102, 186602 (2009).

[23] A. Bauer, M. Garst, and C. Pfleiderer, Phys. Rev. Lett. 110, 177207 (2013).

[24] J. Zang, M. Mostovoy, J. H. Han, and N. Nagaosa, Phys. Rev. Lett. 107, 136804 (2011).

[25] P. Milde, D. Köhler, J. Seidel, L. M. Eng, A. Bauer, A. Chacon, J. Kindervater, S. Mühlbauer, C. Pfleiderer, S. Buhrandt, C. Schütte, and A. Rosch, Science 340, 1076 (2013).

[26] S. S. P. Parkin, M. Hayashi, and L. Thomas, Science 320, 190 (2008).

[27] D. A. Allwood, G. Xiong, C. C. Faulkner, D. Atkinson, D. Petit, and R. P. Cowburn, Science 309, 1688 (2005).

[28] R. Tomasello, E. Martinez, R. Zivieri, L. Torres, M. Carpentieri, and G. Finocchio, Sci. Rep. 4, 6784 (2014).

[29] Y. Zhou and M. Ezawa, Nat. Commun. 5, 4652 (2014).

[30] F. Ma, Y. Zhou, H. B. Braun, and W. S. Lew, Nano Lett. 15, 4029 (2015).

[31] X. Xing and Y. Zhou, NPG Asia Mater. 8, e246 (2016).

[32] X. Zhang, M. Ezawa, and Y. Zhou, Sci. Rep. 5, 9400 (2015).

[33] X. Zhang, Y. Zhou, M. Ezawa, G. P. Zhao, and W. Zhao, Sci. Rep. 5, 11369 (2015).

[34] I. M. Miron, T. Moore, H. Szambolics, L. D. Buda-Prejbeanu, S. Auffret, B. Rodmacq, S. Pizzini, J. Vogel, M. Bonfim, A. Schuhl, and G. Gaudin, Nat. Mater. 10, 419 (2011).
[35] K.-S. Ryu, L. Thomas, S.-H. Yang, and S. Parkin, Nat Nanotechnol. 8, 527 (2013)

[36] S. Emori, U. Bauer, S.-M. Ahn, E. Martinez, and G. S. D. Beach, Nat. Mater. 12, 611 (2013).

[37] A. Brataas, Nat. Nanotechnol. 8, 485 (2013).

[38] X. Zhang, G. P. Zhao, H. Fangohr, J. P. Liu, W. X. Xia, J. Xia, and F. J. Morvan, Sci. Rep. 5, 7643 (2015).

[39] J. Iwasaki, W. Koshibae, and N. Nagaosa, Nano Lett. 14, 4432 (2014).

[40] A. Kunz, Appl. Phys. Lett. 94, 132502 (2009).

[41] A. Thiaville, S. Rohart, E. Jué, V. Cros, and A. Fert, Europhys. Lett. 100, 57002 (2012).

[42] S. Emori, E. Martinez, K.-J. Lee, H.-W. Lee, U. Bauer, S. M. Ahn, P. Agrawal, D. C. Bono, and G. S. D. Beach, Phys. Rev. B 90, 184427 (2014)

[43] O. Boulle, S. Rohart, L. D. Buda-Prejbeanu, E. Jue, I. M. Miron, S. Pizzini, J. Vogel, G. Gaudin, and A. Thiaville, Phys. Rev. Lett. 111, 217203 (2013).

[44] W. Jiang, P. Upadhyaya, W. Zhang, G. Yu, M. B. Jungfleisch, F. Y. Fradin, J. E. Pearson, Y. Tserkovnyak, K. L. Wang, O. Heinonen, S. G. E. te Velthuis, and A. Hoffmann, Science 349, 283 (2015).

[45] J. Iwasaki, M. Mochizuki, and N. Nagaosa, Nat. Nanotechnol. 8, 742 (2013).

[46] S. J. Noh, Y. Miyamoto, M. Okuda, N. Hayashi, and Y. K. Kim, J. Appl. Phys. 111, 07D123 (2012).

[47] H. Y. Yuan and X. R. Wang, Phys. Rev. B 89, 054423 (2014).

[48] H. Y. Yuan and X. R. Wang, Phys. Rev. B 92, 054419 (2015).

[49] H. Y. Yuan and X. R. Wang, Eur. Phys. J. B 88, 214 (2015).

[50] O. Boulle, J. Kimling, P. Warnicke, M. Kläui, U. Rüdiger, G. Malinowski, H. J. M. Swagten, B. Koopmans, C. Ulysse, and G. Faini, Phys. Rev. Lett. 101, 216601 (2008).

[51] M. Eltschka, M. Wötzel, J. Rhensius, S. Krzyk, U. Nowak, M. Kläui, T. Kasama, R. E. Dunin-Borkowski, L. J. Heyderman, H. J. van Driel, and R. A. Duine, Phys. Rev. Lett. 105, 056601 (2010).

[52] C. Burrowes, A. P. Mihai, D. Ravelosona, J. Kim, C. Chappert, L. Villa, A. Marty, Y. Samson, F. Garcia-Sanchez, L. D. BudaPrejbeanu, I. Todosa, E. E. Fullerton, and J. Attane, Nat. Phys. 6, 17 (2010).

[53] S. D. Pollard, L. Huang, K. S. Buchanan, D. A. Arena, and Y. Zhu, Nat. Commun. 3, 1028 (2012).

[54] Y. Zhou, E. Iacocca, A. A. Awad, R. K. Dumas, F. C. Zhang, H B. Braun, and J. Åkerman, Nat. Commun. 6, 8193 (2015).

[55] H. Y. Yuan and X. R. Wang, Sci. Rep. 6, 22638 (2016).

[56] A. Vansteenkiste, J. Leliaert, M. Dvornik, M. Helsen, F. Garcia-Sanchez, and B. van Waeyenberge, AIP Adv. 4, 107133 (2014).

[57] L. D. Landau and E. M. Lifshitz, Phys. Z. Sowjetunion 8, 153 (1935) [reprint in Ukr. J. Phys. 53 (Special issue), 14 (2008)].

[58] T. L. Gilbert, IEEE Trans. Magn. 40, 3443 (2004).

[59] J. Slonczewski, J. Magn. Magn. Mater. 159, L1 (1996).

[60] S. Zhang and Z. Li, Phys. Rev. Lett. 93, 127204 (2004).

[61] P. J. Metaxas, J.-P. Jamet, A. Mougin, M. Cormier, J. Ferré, V. Baltz, B. Rodmacq, B. Dieny, and R. L. Stamps, Phys. Rev. Lett. 99, 217208 (2007)

[62] J.-P. Tetienne, T. Hingant, L. Martínez, S. Rohart, A. Thiaville, L. H. Diez, K. Garcia, J.-P. Adam, J.-V. Kim, J.-F. Roch, I. Miron, G. Gaudin, L. Vila, B. Ocker, D. Ravelosona, and V. Jacques, Nat. Commun. 6, 6733 (2015). 
[63] M. R. Scheinfein, LLG Micromagnetics Simulator (Release LLG v4_x64); available at http://llgmicro.home.mindspring. $\mathrm{com} /$.

[64] M. Pereiro, D. Yudin, J. Chico, C. Etz, O. Eriksson, and A. Bergman, Nat. Commun. 5, 4815 (2015).

[65] T. Okuno, K. Mibu, and T. Shinjo, J. Appl. Phys. 95, 3612 (2004).

[66] A. Thiaville, J. M. García, R. Dittrich, J. Miltat, and T. Schrefl, Phys. Rev. B 67, 094410 (2003).

[67] C. Moutafis, S. Komineas, C. A. F. Vaz, J. A. C. Bland, T. Shima, T. Seki, and K. Takanashi, Phys. Rev. B 76, 104426 (2007).

[68] X. Zhang, Y. Zhou, and M. Ezawa, Nat. Commun. 7, 10293 (2016).

[69] See Supplemental Material at http://link.aps.org/supplemental/ 10.1103/PhysRevB.94.054408 for the evolution of the fractional skyrmion under various conditions.

[70] A. A. Thiele, Phys. Rev. Lett. 30, 230 (1973).

[71] T. Schneider, A. A. Serga, B. Leven, B. Hillebrands, R. L. Stamps, and M. P. Kostylev, Appl. Phys. Lett. 92, 022505 (2008).

[72] K.-S. Lee and S.-K. Kim, J. Appl. Phys. 104, 053909 (2008).
[73] X. Xing, Y. Yu, S. Li, and X. Huang, Sci. Rep. 3, 2958 (2013).

[74] S. Breitkreutz, I. Eichwald, J. Kiermaier, G. Hiblot, G. Csaba, W. Porod, D. Schmitt-Landsiedel, and M. Becherer, J. Appl. Phys. 115, 17D506 (2014).

[75] H. Y. Yuan and X. R. Wang, J. Magn. Magn. Mater. 368, 70 (2014).

[76] V. V. Kruglyak, S. O. Demokritov, and D. Grundler, J. Phys. D: Appl. Phys. 43, 264001 (2010).

[77] B. Lenk, H. Ulrichs, F. Garbs, and M. Munzenberg, Phys. Rep. 507, 107 (2011).

[78] A. Khitun, M. Bao, and K. L. Wang, J. Phys. D: Appl. Phys. 43, 264005 (2010).

[79] I. M. Miron, G. Gaudin, S. Auffret, B. Rodmacq, A. Schuhl, S. Pizzini, J. Vogel, and P. Gambardella, Nat. Mater. 9, 230 (2010).

[80] L. Liu, C. F. Pai, Y. Li, H. W. Tseng, D. C. Ralph, and R. A. Buhrman, Science 336, 555 (2012).

[81] A. V. Khvalkovskiy, V. Cros, D. Apalkov, V. Nikitin, M. Krounbi, K. A. Zvezdin, A. Anane, J. Grollier, and A. Fert, Phys. Rev. B 87, 020402(R) (2013). 